\title{
Properties of a charge in the nanoheterosystems with quantum wells and barriers
}

\author{
V.I. Boichuk, I.V. Bilynskyi, I.O.Shakleind;, V.B. Holskyi \\ Department of Theoretical Physics, Ivan Franko Drohobych State Pedagogical University,
} 24 Ivan Franko Str., 82100 Drohobych, Ukraine

Received December 11, 2008, in final form August 28, 2009

\begin{abstract}
In the paper a quasi-one-dimensional three-layer nanowire (NW) with an intermediate layer at the separation boundaries is described by the Kronig-Penney model with $\delta$-function potentials. The distance between the last or supreme atoms of the intermediate layer is a parameter of the problem and ranges from zero to two lattice parameters of crystals. A precise solution is obtained for the quantum-mechanical reflection coefficient $R$ which makes it possible to determine the dependence of the factor on a wave vector, widths of an intermediate layer and a monolayer of the nanoheterostructure. The specific calculations are performed for the GaAs/AIAs/GaAs and AlAs/GaAs/AIAS nanowires. The comparison of the reflection coefficients in the envelope-function approximation and in the effective mass method is performed at small values of a wave vector. It is shown that similar results can be received using the refined procedure, originally proposed by Harrison, with appropriately taken parameters. For the cosinusoidal dependence of the energy on a wave vector which arises in the Kronig-Penney model within the framework of the S-matrix scattering method, we determined the binding states energy for a AlAs/GaAs/AIAs quantum well wire.
\end{abstract}

Key words: heterostructure, intermediate layer, Kronig-Penney model, reflection coefficient, S-matrix scattering method

PACS: $71.55 .-i, 73.21 . L a, 79.60 . J V$

\section{Introduction}

Recently various nanostructures have been intensively studied both experimentally and theoretically due to their interesting electric and optical properties. A number of works is devoted to the heterostructures with interface planes GaAs/AlAs in which the layers of AlAs are placed in crystals GaAs or vice versa [1 4]. Electronic properties of such heterostructures are associated with electrons of the $\Gamma$-valley of the band spectrum. The problem of electron transmission through a boundary of different crystals arises in heterojunction electronics and in transport properties of polycrystalline materials [5 10]. In order to solve the problem precisely, it is necessary to know the exact Bloch functions of constituent materials and the evanescent states near the interface. In many works the Bloch functions are replaced with the envelope ones and the effective mass approximation is used [5 9]. It is usual to assume that the envelope function and its derivatives need to be continuous across the interface [10 13. In principle, these conditions would follow from a valid expression for the current operator in terms of the envelope functions. Such an expression would depend on the proper form of the effective kinetic energy operator acting on the envelope functions - as well as be correct in the vicinity of the interfaces where the band structure changes abruptly. Although the expression of this operator was discussed in [5, 14 16], it has never been generally derived from the first principles. Instructive results, however, have been obtained in theories based on the Kane $\vec{k} \cdot \vec{p}$ model and tight binding approximation [14 16]. Harrison in [5] used the continuity conditions of $\xi \Phi$ and $(m \xi)^{-1} \nabla \Phi$, where $\Phi$ is an envelope function, $m(x)$ is an effective mass, $\xi(x)$ is a scalar parameter which is hopefully not too strongly dependent on the energy near the band edge in each crystal. For one-dimensional crystals, the Harrison conditions in their most general form $(\beta \Phi$ and $\alpha(\partial \Phi / \partial x)$ are continuous) can be justified by the fact that the effective Schrödinger

*E-mail: ioshak@mail.ru 
equations, defining the envelope functions in each crystal, are second-order differential equations. Hence, their solutions can be matched with two parameters, $\alpha$ and $\beta$. The continuity of current density requires that $\alpha \beta m$ be continuous across the interface. Thus, the matching problem reduces to one unknown parameter $(\xi)$. In most publications on the problem of choice and substantiation of boundary conditions, the authors studied the heterostructures containing one interface at which all the values, and the distance between atoms in particular, change abruptly.

Recent developments in nanotechnologies have led to the publication of a number of experimental studies of physical properties of quantum wires. Significant progress in electronic microscopy makes it possible to experimentally investigate the structure of grown quantum wires, observe the size and form dependences of these quasi-one-dimensional nanoscale heterosystems on their growth conditions. In particular, for the InGaAs, GaAs, $\mathrm{ZnO}$ quantum wires carrier concentrations and mobilities with different external physical parameters are defined, and luminescence, electro- and photoconductivity measurements are done [19, 20].

The fabrication of axial heterostructure in the direction of quantum wire axis by means of alternating semiconductor materials with various energy gaps substantially widens the potential application of nanowires. In this way resonant diodes and quantum transistors used in novel nanoelectronic devices are made. Hence, in plenty of experimental as well as theoretical works, electron, hole, and exciton energy states are studied in composite quantum wires [10 13, 17 20].

The purpose of the present work is to develop the theory of reflection coefficient in an infinite quasi-one-dimensional three-layer wire (with quantum well or quantum barrier) with account of an intermediate layer at the interfaces in the Kronig-Penney model with $\delta$-function potentials peaks. In the intermediate layer the distance between adjacent atoms can range from zero to two lattice parameters. We also investigate the dependence of reflection coefficient on the wave vector, the thickness of an intermediate layer, width of a quantum well (a barrier) of the heterostructure and define the bound state energies by the S-matrix scattering method.

\section{A heterostructure model. General theory}

A model of the quantum wire which consists of three regions is presented in figure 1. The left "crystal" (labeled I) and the right one (labeled III) are identical half-infinite quasi-one-dimensional crystals. The internal regions (labeled II) correspond to the quantum well or barrier of the heterosystem. All three crystalline regions represent arrays of $\delta$-functions of strength $P_{i}$ and periodicity $d_{i}(i=1,2)$. The size of $\delta$-function peaks is different in the first (third) and second regions. In addition, one can have a potential discontinuity at the interface plane. This permits us to isolate the band-structure effects from those associated with a potential step at the interface. An important characteristic of the boundaries is the size $b=b_{1}+b_{2}$ of the intermediate layers containing "atoms" of both crystals. This is a source of uncertainty even in our model problem because the reflection coefficient and particle energy will be a strong function of $b$ since the intermediate layers break the translation symmetry. We shall not restrict ourselves to any specific value of $b$. For simplicity of presentation we shall assume that the gap (the intermediate layer) is not too large: $b<d_{1}+d_{2}$.

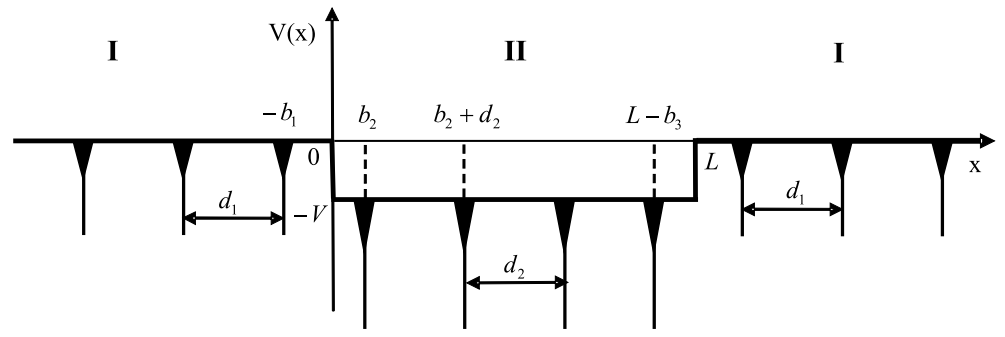

Figure 1. A heterosrtucture model.

Then we can always choose the crystal unit cells at the left interface so that they abut at the point $x=0$ without a gap or overlap. Then the Schrödinger equation of an electron for 
$-b_{1}-d_{1}<x<b_{2}+d_{2}$ is written as

$$
\left\{\frac{-\hbar^{2}}{2 m_{0}} \frac{d^{2}}{d x^{2}}-P_{i} \delta\left(x \pm b_{i}\right)+V_{i}\right\} \Psi_{k_{i}}^{(i)}=E \Psi_{k_{i}}^{(i)}, \quad i=1,2,
$$

where $m_{0}$ is a free electron mass. In equation (1) $i=1$ is used in the first region, while $i=2$ corresponds to the second region, thus $V_{1}=0, V_{2}=-V$. Similar equations can be written for an electron at the interface $x=L$.

The first and the third region are half-infinite, that is why the functions $\Psi_{k_{1}}^{(1)}$ and $\Psi_{k_{1}}^{(3)}$ are the Bloch functions which satisfies the relation

$$
\Psi_{k_{1}}^{(1)}(0)=\mathrm{e}^{\mathrm{i} k_{1} d_{1}} \Psi_{k_{1}}^{(1)}\left(-d_{1}\right), \quad \Psi_{k_{1}}^{(3)}(L)=\mathrm{e}^{-\mathrm{i} k_{1} d_{1}} \Psi_{k_{1}}^{(3)}\left(L+d_{1}\right) .
$$

Similar relations can be written for the wave-function derivatives. Note that at the pole points of the Dirac $\delta$-function the wave-function derivative changes stepwise [17]. In particular

$$
\begin{aligned}
\Psi_{k_{1}}^{(1)^{\prime}}\left(-b_{1}+0\right)-\Psi_{k_{1}}^{(1)^{\prime}}\left(-b_{1}-0\right) & =-\frac{2 m_{0} P_{1}}{\hbar^{2}} \Psi_{k_{1}}^{(1)}\left(-b_{1}\right), \\
\Psi_{k_{1}}^{(3)^{\prime}}\left(L+b_{1}+0\right)-\Psi_{k_{1}}^{(3)^{\prime}}\left(L+b_{1}-0\right) & =-\frac{2 m_{0} P_{1}}{\hbar^{2}} \Psi_{k_{1}}^{(3)}\left(L+b_{1}\right) .
\end{aligned}
$$

The use of translational properties of the wave functions makes it possible to obtain the dispersion equation of an electron in the first and third regions

$$
\cos \left(k_{1} d_{1}\right)=\cos \left(q_{1} d_{1}\right)-U_{1} \frac{\sin \left(q_{1} d_{1}\right)}{q_{1} d_{1}} .
$$

where $U_{1}=\frac{m_{0} P_{1} d_{1}}{\hbar^{2}}, q_{1}=\sqrt{\frac{2 m_{0} E}{\hbar^{2}}}, k_{1}$ is an electron wave vector.

The total wave functions in the first and third regions are as follows:

$$
\begin{aligned}
& \Psi_{1}(x)=\Psi_{k_{1}}^{(1)}(x)+r \Psi_{-k_{1}}^{(1)}(x), \\
& \Psi_{3}(x)=t \Psi_{k_{1}}^{(3)}(x) .
\end{aligned}
$$

In the vicinity of the interfaces the electron wave functions with a certain wave vector can be written

$$
\begin{aligned}
& \Psi_{k_{1}}^{(1)}(x)=A_{k_{1}}\left[\frac{1-\mathrm{e}^{-\mathrm{i}\left(q_{1}+k_{1}\right) d_{1}}}{1-\mathrm{e}^{-\mathrm{i}\left(q_{1}-k_{1}\right) d_{1}}} \mathrm{e}^{\mathrm{i} q_{1}\left(x+b_{1}\right)}+\mathrm{e}^{-\mathrm{i} q_{1}\left(x+b_{1}\right)}-\left(1-\mathrm{e}^{-\mathrm{i}\left(q_{1}+k_{1}\right) d_{1}}\right) \mathrm{e}^{\mathrm{i} q_{1}\left|x+b_{1}\right|}\right], \\
& \Psi_{k_{1}}^{(3)}(x)=A_{k_{1}}\left[\frac{1-\mathrm{e}^{-\mathrm{i}\left(q_{1}+k_{1}\right) d_{1}}}{1-\mathrm{e}^{-\mathrm{i}\left(q_{1}-k_{1}\right) d_{1}}} \mathrm{e}^{\mathrm{i} q_{1}\left(x-L-b_{1}\right)}+\mathrm{e}^{-\mathrm{i} q_{1}\left(x-L-b_{1}\right)}-\left(1-\mathrm{e}^{-\mathrm{i}\left(q_{1}+k_{1}\right) d_{1}}\right) \mathrm{e}^{\mathrm{i} q_{1}\left|x-L-b_{1}\right|}\right] .
\end{aligned}
$$

For other points of space the functions $\Psi_{k_{1}}^{(1)}(x)$ and $\Psi_{k_{1}}^{(3)}(x)$ can be found from the transcendent conditions imposed on the wave function.

In the second region of the heterosystem it is also possible to find the wave function $\Psi_{2}(x)$ which describes a particle. Its form is rather complicated and depends on the number of atoms $N$ of the region (see Appendix).

From the matching conditions of functions $\Psi_{1}(x), \Psi_{2}(x)$ and $\Psi_{3}(x)$ at $x=0, x=L$

$$
\begin{array}{cl}
\Psi_{1}(0)=\Psi_{2}(0), & \Psi_{2}(L)=\Psi_{3}(L), \\
\left.\Psi_{1}^{\prime}(x)\right|_{x=0}=\left.\Psi_{2}^{\prime}(x)\right|_{x=0}, & \left.\Psi_{2}^{\prime}(x)\right|_{x=L}=\left.\Psi_{3}^{\prime}(x)\right|_{x=L}
\end{array}
$$

one can define the reflection amplitude $r$. Once $r$ is known, we can determine the coefficients of reflection $(R)$ and transmission $(T)$ of a charge in the heterostructure

$$
R=|r|^{2}, \quad T=1-R .
$$


If the 2nd region of the heterostructure contains a great number of atoms $(N \gg 1)$, it is possible to neglect the absence of translation symmetry and use the Kronig-Penney model. Then the wave function of the first cell in the second layer can be written in the form of (5) provided $q_{1}$ is substituted with $q_{2}=\sqrt{\frac{2 m_{0}(E+V)}{\hbar^{2}}}$, and $k_{1}$ with $k_{2}$ respectively.

The wave function of the last cell can be found using the translative property of the Bloch function. The total wave function in this layer is represented by

$$
\Psi_{2}(x)=p \Psi_{k_{2}}^{(2)}(x)+f \Psi_{-k_{2}}^{(2)}(x) .
$$

Using the matching conditions of functions (7), one can find the expression for the reflection coefficient amplitude $r$

$$
r=\frac{\Psi_{k_{1}}^{(1)}(0)\left[\mu \Psi_{k_{2}}^{(2)^{\prime}}(0)+\Psi_{-k_{2}}^{(2)^{\prime}}(0)\right]-\Psi_{k_{1}}^{(1)^{\prime}}(0)\left[\mu \Psi_{k_{2}}^{(2)}(0)+\Psi_{-k_{2}}^{(2)}(0)\right]}{\Psi_{-k_{1}}^{(1)^{\prime}}(0)\left[\mu \Psi_{k_{2}}^{(2)}(0)+\Psi_{-k_{2}}^{(2)}(0)\right]-\Psi_{-k_{1}}^{(1)}(0)\left[\mu \Psi_{k_{2}}^{(2)^{\prime}}(0)+\Psi_{-k_{2}}^{(2)^{\prime}}(0)\right]},
$$

where the following notation is used:

$$
\mu=\frac{\Psi_{-k_{2}}^{(2)^{\prime}}(0) \Psi_{k_{1}}^{(3)}(L)-\Psi_{-k_{2}}^{(2)}(0)\left(d_{2}\right) \Psi_{k_{1}}^{(3)^{\prime}}(L)}{\Psi_{k_{2}}^{(2)}(0) \Psi_{k_{1}}^{(3)^{\prime}}(L)-\Psi_{k_{2}}^{(2)^{\prime}}(0) \Psi_{k_{1}}^{(3)}(L)} \mathrm{e}^{-2 \mathrm{i} k_{2} L}
$$

The reflection coefficient obtained due to (10) can be compared to its value from equation (7) and expressions (D.5)-(D.8). Then we can establish the criteria of the use of the infinite crystal approach in the 2nd layer of the heterostructure.

The Bloch wave functions of the crystals which form real heterostructures are unknown. Therefore, as a rule, the envelope-function model is used. In our case the envelope function looks like

$$
\Phi(x)=\left\{\begin{array}{l}
\Phi_{1}(x)=\mathrm{e}^{\mathrm{i} k_{1} x}+r \mathrm{e}^{-\mathrm{i} k_{1} x}, \quad x<0 \\
\Phi_{2}(x)=A \mathrm{e}^{\mathrm{i} k_{2} x}+B \mathrm{e}^{-\mathrm{i} k_{2} x}, \quad 0<x<L \\
\Phi_{3}(x)=t \mathrm{e}^{\mathrm{i} k_{1}(x-L)}, \quad x>L
\end{array}\right.
$$

Functions $\Phi_{i}(x)(i=1,2,3)$ represent linear combinations of plane-wave solutions of the effective-mass Hamiltonians,

$$
H^{(i)}=E_{n}^{(i)}-\frac{\hbar^{2}}{2 m_{i}} \frac{\partial^{2}}{\partial x^{2}}, \quad i=1,2,3
$$

describing electronic motion near the band edges $E_{n}^{(i)}$ in each crystal. Boundary conditions of envelope functions are frequently based on the continuity condition of $\Phi$ and $\frac{1}{m} \frac{\partial \Phi}{\partial x}[10-14,19-21]$.

More general conditions which are imposed on envelope functions are used by Harrison [5]

$$
\begin{aligned}
\beta_{1} \Phi_{1}(0) & =\beta_{2} \Phi_{2}(0), \\
\beta_{2} \Phi_{2}(L) & =\beta_{1} \Phi_{3}(L), \\
\left.\alpha_{1} \frac{\partial \Phi_{1}}{\partial x}\right|_{x=0} & =\left.\alpha_{2} \frac{\partial \Phi_{1}}{\partial x}\right|_{x=0}, \\
\left.\alpha_{2} \frac{\partial \Phi_{2}}{\partial x}\right|_{x=L} & =\left.\alpha_{1} \frac{\partial \Phi_{3}}{\partial x}\right|_{x=L} .
\end{aligned}
$$

Parameters $\alpha_{i}$ and $\beta_{i}(i=1,2)$ define physical conditions at the interface. They (or part of them) are considered matching parameters of the problem.

Continuity of the flux (in the effective-mass approximation) in this case requires that

$$
\alpha_{1} \beta_{1} m_{1}=\alpha_{2} \beta_{2} m_{2} \text {. }
$$


Using (13) and (14), we determine the reflection coefficient for the heterostructure

$$
R=\frac{\frac{\xi_{1}^{2}}{\xi_{2}^{2}}\left[\frac{m_{2}^{2} k_{1}^{2} \xi_{2}^{2}}{m_{1}^{2} k_{2}^{2} \xi_{1}^{2}}-1\right]^{2} \sin ^{2} k_{2} L}{4 \frac{m_{2}^{2} k_{1}^{2}}{m_{1}^{2} k_{2}^{2}}+\frac{\xi_{1}^{2}}{\xi_{2}^{2}}\left[\frac{m_{2}^{2} k_{1}^{2} \xi_{2}^{2}}{m_{1}^{2} k_{2}^{2} \xi_{1}^{2}}-1\right]^{2} \sin ^{2} k_{2} L}, \quad \frac{\beta_{1}^{2}}{\beta_{2}^{2}}=\frac{\xi_{1}}{\xi_{2}} .
$$

The expressions of the wave function allow us to obtain the energies of the quantum wire. We consider the composite nanowire which contains a quantum well in the second region. In this case there should exist particle binding states. The energy of these states is obtained by the S-matrix scattering method [22].

Using the S-matrix scattering method, we can express the particle wave function in the first layer in the form

$$
\Psi_{1}(x)=A\left(\Psi_{-k_{1}}^{(1)}(x)-S(E) \Psi_{k_{1}}^{(1)}(x)\right) .
$$

Using the matching conditions of functions $(7), S(E)$ is written

$$
S(E)=-r^{-1}(E) \text {. }
$$

In order to find discrete particle energy values in the heterostructure, it is necessary to find the S-matrix poles.

\section{Specific calculations. The analysis of the obtained results}

We apply the present model to the study of GaAs/AlAs/GaAs and AlAs/GaAs/AlAS quantum wire. For this purpose, once we know the effective masses, lattice parameters and a potential step, it is necessary to determine parameters of the problem: $P_{1}, P_{2}, d_{1}, d_{2}$.

From dispersion relation (4) one defines extreme energies in the limit $k_{i} \rightarrow 0$. If in the heterosystem at the interface there are no additional fields, and the electron potential energy does not change $\left(V_{2}=0\right)$, then for both crystals $(i=1,2)$ the extreme energies are found from the following equations:

$$
\begin{gathered}
\operatorname{tg} \sqrt{\frac{m_{0} d_{i}^{2}}{2 \hbar^{2}} E_{n}}=-\frac{U_{i}}{\sqrt{\frac{m_{0} d_{i}^{2}}{2 \hbar^{2}} E_{n}}}, \quad n=1,3,5, \ldots, \\
\sin \sqrt{\frac{m_{0} d_{i}^{2}}{2 \hbar^{2}} E_{n}}=0, \quad n=2,4,6, \ldots
\end{gathered}
$$

One can also derive effective masses of odd and even energy bands

$$
\begin{gathered}
\frac{m_{n}}{m_{0}}=-\frac{2 \hbar^{2} U_{i}}{m_{0} d_{i}^{2} E_{n}}\left[1-\frac{2 U_{i}}{\frac{m_{0} d_{i}^{2}}{2 \hbar^{2}} E_{n}+U_{i}^{2}}\right], \quad n=1,3,5, \ldots \\
\frac{m_{n}}{m_{0}}=-\frac{U_{i}}{\pi^{2} n^{2}}, \quad n=2,4,6, \ldots
\end{gathered}
$$

From equation (4) we obtained the dispersion relation for crystals GaAs and AlAs $\left(d_{1}=5.653 \dot{A}\right.$, $m=0.066 m_{0}-\mathrm{GaAs} ; d_{2}=5.66 \dot{A}, m=0.124 m_{0}-\mathrm{AlAs}, V_{0}=570 \mathrm{meV}$ is a misalignment between third bands). The spectrum under consideration is realized providing that

$$
U_{1}=\frac{\hbar^{2}}{m_{0} P_{1} d_{1}}=-3.01, \quad U_{2}=\frac{\hbar^{2}}{m_{0} P_{2} d_{2}}=-4.79 .
$$

Having the necessary parameters, it is possible to determine the coefficient of reflection $R$ or transmission $T$.

Reflection and transmission of electrons in such NW's essentially depend on the size of the intermediate layer (boundary), that is $b=b_{1}+b_{2}$, which results from the distortion of lattice 

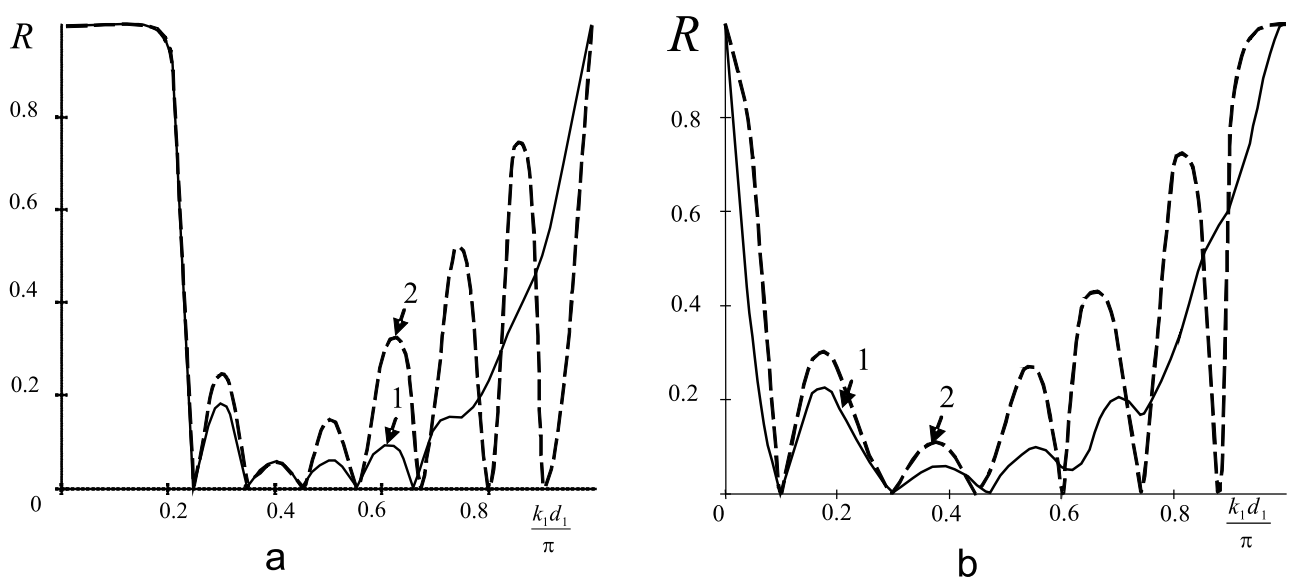

Figure 2. The electron reflection coefficient of a GaAs/AlAs/GaAs (a) and AlAs/GaA/AlAs (b) three-layer system. $U_{1}=-3.01, U_{2}=-4.79$. Curve 1 corresponds to $b=b_{1}+b_{2}=d_{2}$ $\left(b_{1}=0.1 d_{2}, b_{2}=0.9 d_{2}\right)$. Curve 2 corresponds to $b=b_{1}+b_{2}=1.8 d_{2}\left(b_{1}=0.1 d_{2}, b_{2}=1.7 d_{2}\right)$.

parameters near the interface of the heterostructure. The change of these values leads to the change of local maxima in the dependence $R=R\left(k_{1}\right)$ as well as to their shift.

In figure 2 the dependence of the reflection coefficient $R$ on the dimensionless wave vector of an electron incident on a quantum well (barrier) at a varying size of the intermediate layer is shown for the GaAs/AlAs/GaAs and AlAs/GaAs/AlAs quasi-one-dimensional three-layer heterosystems.

For the first heterostructure (figure 2 (a)), in contrast to the second system the dependence $R=R\left(k_{1}\right)$ consists of two regions - scattering under the barrier and above it (for $\frac{k_{1} d_{1}}{\pi}<0,2$ electron transmission is not practically observed). However, in general, the wave-vector dependence of the reflection coefficient $R=R\left(k_{1}\right)$ for different sizes of the intermediate layer has identical behavior for both heterostructure types. At small $\mathrm{k}_{1}$ the reflection coefficient is close to unity and decreases as the wave vector rises. Each structure exhibits specific points at which perfect transmission $(R=0)$ is observed. It is noteworthy that the number of maxima (and accordingly, perfect transmission points) is larger for the GaAs/AlAs/GaAs nanowire in spite of the fact, that a transmission interval of this system is much smaller than that of AlAs/GaAs/AlAs. The sizes of the corresponding maxima differ little for both crystal types. At $\frac{k_{1} d_{1}}{\pi}=1$ the reflection coefficient reaches its maximum possible value $(R=1)$.

For both heterostructures, the results $R=R\left(k_{1}\right)$ obtained by exact and approximated solutions are compared. At large sizes of the second region $R=R\left(k_{1}\right) L\left(L \geqslant 4 d_{2}\right)$ both expressions yield qualitatively similar results. However, a better agreement is obtained for the GaAs/AlAs/GaAs system.

It can be concluded that for composite NW's in the limit of quantum well or barrier widths larger than $4 d_{2}$ an electron can be described by the Bloch function.

Let us analyze the reflection coefficient received within the envelope-function approach and the effective mass approximation refined by the Harrison boundary conditions. We consider a special case of the surface when the intermediate region containing atoms of both crystals is of the size $b=\frac{d_{1}+d_{2}}{2}$. In this case one can estimate the exact values of matching parameters $\xi_{i} \equiv \beta_{i}^{2}, i=1,2$ through the crystal parameters and the electron energy at the bottom of the band [8]

$$
\xi_{1,2}=\frac{U_{1,2}}{\left(q_{n} d_{1,2}\right)^{2}} \frac{m_{0}}{m_{1,2}}, \quad q_{n}=\sqrt{\frac{2 m_{0} E_{n}}{\hbar^{2}}} .
$$

If in (21) one takes into account the expression for the effective mass (20), one can see that for the higher-lying bands, when $q_{n} d_{1,2} \gg\left|U_{1,2}\right|$, values of $\xi_{i}$ tend to unity, and formula (18) is transferred into formula (15) of the reflection coefficient. It can be obtained by the envelopefunction approach $\left(\frac{\xi_{1}}{\xi_{2}}=1\right)$. For the heterosystem models under consideration the following exact 
values were obtained: $\xi_{1}=0,91, \xi_{2}=0,768$, which, in its turn, makes it possible to compare the results received by the effective mass approximation with the exact solution.

In figure 3 (a) the reflection coefficient $R=R\left(k_{1}\right)$ for the AlAs/GaAs/AlAs heterosystem, calculated according to the exact solution and effective mass approximation refined by Harrison boundary conditions (15), is shown for different barrier sizes. One can see that in the limit of small wave-vector values $k_{1} \rightarrow 0$, where the effective-mass approach is applicable, a good qualitative agreement of the results is observed. A further wave-vector increase leads to the shift of points of perfect electron transmission $(R=0)$ in the direction of bigger $k_{1}$ and a certain disagreement of maxima.
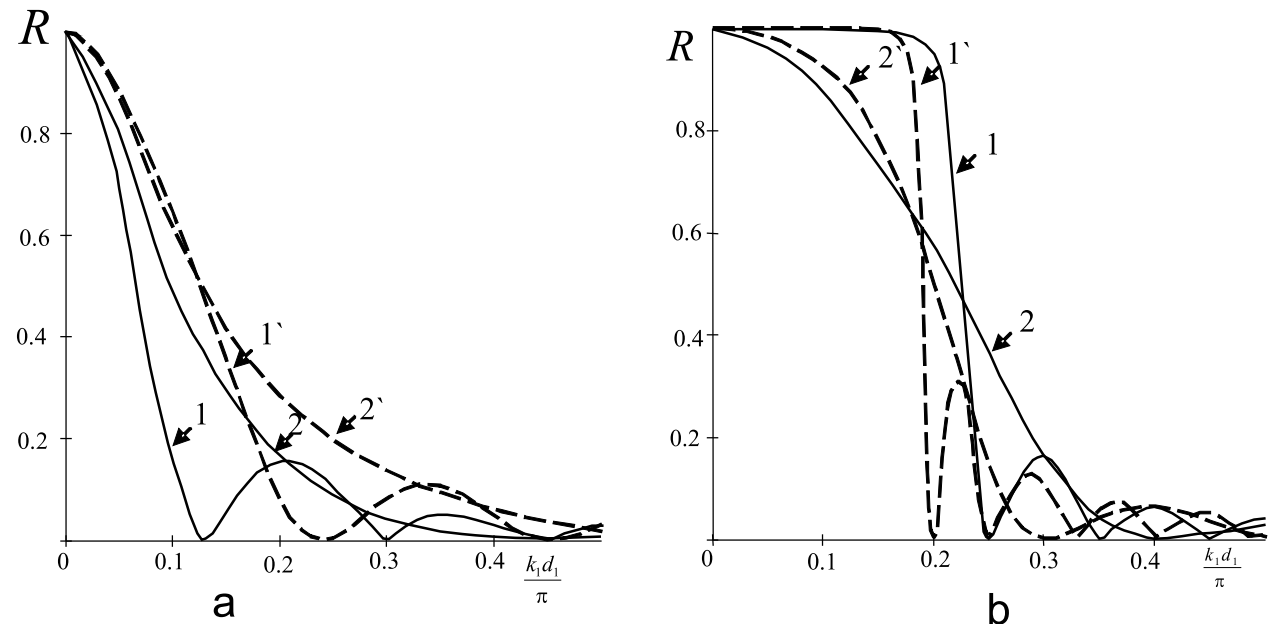

Figure 3. The electron reflection coefficient of a AlAs/GaAs/AlAs (a) and AlAs/GaA/AlAs (b) three-layer system. A solid curve stands for an accurate solution, dashed curve stands for the effective-mass method specified by Harrison conditions. Curves 1 and $1^{\prime}$ correspond to $\mathrm{L}=8 d_{2}$ (45.224 A); curves 2 and $2^{\prime}$ correspond to $\mathrm{L}=2 d_{2}(11.306 \mathrm{~A})$.

The calculations performed for the GaAs/AlAs/GaAs heterostructure (figure 3 (b)) have also shown a quite good qualitative agreement of the results obtained by the effective-mass approximation with the Harrison matching conditions and the exact solution. In the limit $k_{1} \rightarrow 0$, both methods yield almost identical results. With a further wave vector increase, the divergence becomes more essential. This can be assigned to the fact that the effective mass approximation works only in the interval of small wave-vector values. Note that for the heterostructure under consideration, in contrast to the structure AlAs/GaAs/AlAs, with an increase of the wave vector one can observe the shift of perfect electron transmission points $(R=0)$ in the direction of smaller $k_{1}$ received in the effective mass approximation. It is accompanied by a more essential disagreement of maxima. However, it is necessary to mention that this divergence is much smaller than that when we compare the exact solution and the results obtained in the effective-mass approximation in case of $\xi_{1,2}=1$.

As is seen from the plots, with the reduction of a quantum well (barrier) size the mean value of the electron transmission coefficient increases which, in its turn, is expected due to physical reasons.

Therefore, one can come to the conclusion that for the considered surface type, which corresponds to the heterostructures with close lattice parameters, the Harrison matching conditions are applicable and yield good results.

Using the S-matrix scattering method, the energy of binding states is determined for the AlAs/GaAs/AlAs quantum well nanowire. According to this procedure, the state energy is set by those energy values which are the S-matrix scattering poles. This problem is far from being simple because of complex numerical calculations. An analytical expression of the S-matrix (especially of an exact expression of the wave function in the second layer) is rather complicated. Besides, 
the values of the functions $k_{i}=k_{i}(E)(i=1,2)$ are found from the transcendental equations for wave vectors.

The results of calculations of the binding state energy in a quantum well which is formed by third bands for $L=4 d_{2}$ are presented in figure 4 . One can see that at a fixed value of $b_{2}=d_{2} / 2$ the energy depends on $b_{1}$ and therefore, on the distance between the abutting atoms at the interfaces. An increase of $b_{1}$ results in the reduction of the state energy which can be explained by an effective increase of the quantum well size. Besides, one can see that the exact solution (solid line) makes it possible to receive slightly smaller energies than those in the case of the approximate solution (dashed lines), though the difference between the energies is small.

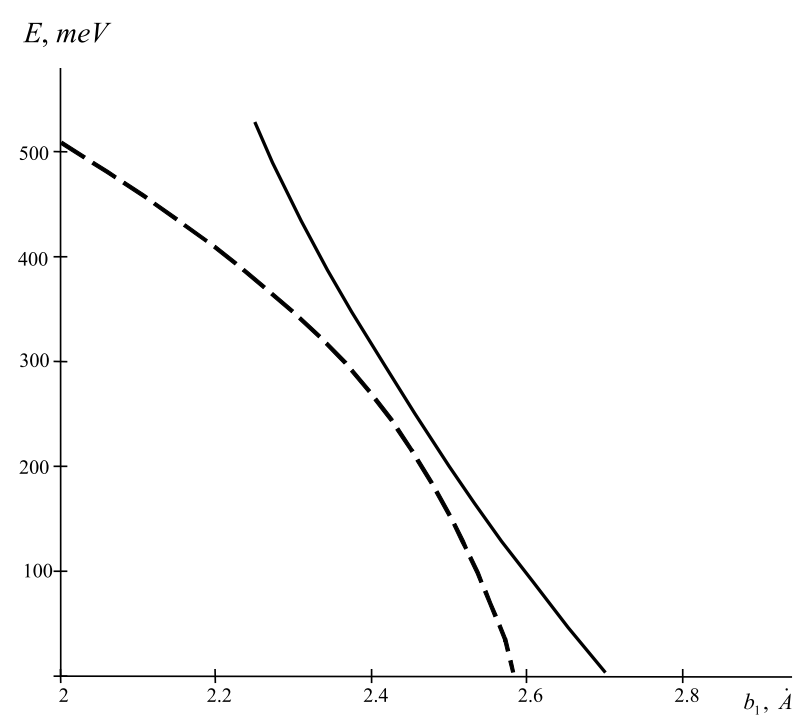

Figure 4. The dependence $E\left(b_{1}\right)$ for the AlAs/GaAs/AlA heterostructure (a third band, $\mathrm{L}=4 d_{2}$ ).

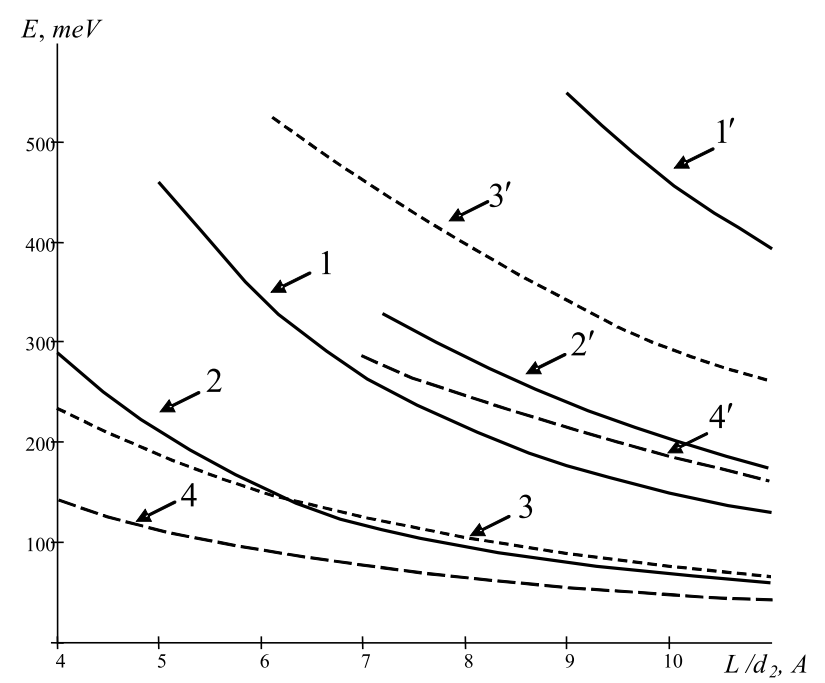

Figure 5. The dependence $E=E(L)$ for the AlAs/GaAs/AlAs heterostructure. Curves $1,1^{\prime}, 2,2^{\prime}$ are obtained by S-matrix scattering method, curves $3,3^{\prime}, 4,4^{\prime}$ - by effective-mass approximation: $1,1^{\prime}, 3,3^{\prime}-\mathrm{E}=\mathrm{E}(\mathrm{L})$ for a third band $2,2^{\prime}, 4,4^{\prime}-E=E(L)$ for a second band.

The dependence of the binding state energy on the quantum well size in the second and third bands is shown in figure 5 . The third bands are characterized by a larger misalignment at the 
interface than the second ones. The energy of the binding states calculated from the bottom or top of the corresponding bands in the 2nd layer is greater for the 3rd band. The increase of quantum well size gives rise to new binding states and a particle monotonous energy reduction obtained both by the S-matrix scattering method (solid lines) and the effective-mass approximation (dashed lines). The derived results are in a good agreement with those in publication [13].

Within the quantum well, the ground state (lines 1,2,3,4) appears at smaller values of $\mathrm{L}$ than the excited state (lines $1^{\prime}, 2^{\prime}, 3^{\prime}, 4^{\prime}$ ) both for the third and second energy bands. The comparison of particle energies obtained by different procedures shows that the energies obtained by the S-matrix scattering method are greater than the corresponding energies in the effective-mass approximation. In particular, for $L=6 d_{3}$, the difference of ground state energies obtained by different methods makes $200 \mathrm{meV}$ for third bands and $90 \mathrm{meV}$ for second ones.

Thus, in the paper a quasi-one-dimensional three-layer nanoheterostructure with an intermediate layer at the interfaces is described by the Kronig-Penney model with $\delta$-function potentials. The distance between the last or supreme atoms of the intermediate layer is a parameter of the problem and ranges from zero to two lattice parameters of crystals. The exact solution is obtained for the reflection coefficient $R$ which allows us to determine the wave-vector dependence of the coefficient, intermediate layer size and quantum well size of the nanowire. The specific calculations performed for the GaAs/AlAs/GaAs and AlAs/GaAs/AlAs heterosystems showed that the envelope-functions and effective-mass approximations for small wave-vector values yield quite good results provided the Harrison matching conditions are used with appropriately taken parameters.

Using the S-matrix scattering method, the energy of binding states is determined for the AlAs/GaAs/AlAs quantum well nanowire. The dependence of the particle energy on the intermediate layer and second layer sizes (quantum well width) is obtained.

\section{Appendix}

The Schrödinger equation for a boundary cell at $x=0$ has the form

$$
\left\{\frac{-\hbar^{2}}{2 m_{0}} \frac{\mathrm{d}^{2}}{\mathrm{~d} x^{2}}-P_{i} \delta\left(x \pm b_{1}\right)\right\} \Psi_{k_{i}}^{(i)}=E \Psi_{k_{i}}^{(i)} .
$$

A general form of the particle wave function which is described by the wave vector $k_{1}$, for the layer containing an atom with the coordinate $x=-b_{1}$, is as follows:

$$
\Psi_{k_{1}}^{(1)}(x)=A \mathrm{e}^{-\mathrm{i} q_{1}\left(x+b_{1}\right)}+B \mathrm{e}^{\mathrm{i} q_{1}\left(x+b_{1}\right)}+C \mathrm{e}^{\mathrm{i} q_{1}\left|x+b_{1}\right|} .
$$

Using the well-known condition applied on the wave function at $x=-b_{1}$, we get

$$
\Psi_{k_{1}}^{(1)^{\prime}}\left(-b_{1}+0\right)-\Psi_{k_{1}}^{(1)^{\prime}}\left(-b_{1}-0\right)=-\frac{2 m_{0} P_{1}}{\hbar^{2}} \Psi_{k_{1}}^{(1)}\left(-b_{1}\right)
$$

$C=-\frac{A_{1}+B_{1}}{1+\frac{i q_{1} d_{1}}{U_{1}}}$, where $U_{1}=\frac{m_{0} P_{1} d_{1}}{\hbar^{2}}$.

From

$$
\Psi_{k_{1}}^{(1)}\left(-d_{1}\right)=\mathrm{e}^{-\mathrm{i} k_{1} d_{1}} \Psi_{k_{1}}^{(1)}(0),\left.\quad \frac{\mathrm{d} \Psi_{k_{1}}^{(1)}}{\mathrm{d} x}\right|_{x=-d}=\left.\mathrm{e}^{-\mathrm{i} k_{1} d_{1}} \frac{\mathrm{d} \Psi_{k_{1}}^{(1)}}{\mathrm{d} x}\right|_{x=0}
$$

we obtain the following relation between the coefficients:

$$
C=-A_{1}\left(1-\mathrm{e}^{-\mathrm{i}\left(q_{1}+k_{1}\right) d_{1}}\right), \quad B=A_{1} \frac{1-\mathrm{e}^{-\mathrm{i}\left(q_{1}+k_{1}\right) d_{1}}}{1-\mathrm{e}^{-\mathrm{i}\left(q_{1}-k_{1}\right) d_{1}}} .
$$

Hence, the normalizing wave function of a particle in the extreme cells of the first and third layers is written

$$
\begin{aligned}
& \Psi_{k_{1}}^{(1)}(x)=A_{k_{1}}\left[\frac{1-\mathrm{e}^{-\mathrm{i}\left(q_{1}+k_{1}\right) d_{1}}}{1-\mathrm{e}^{-\mathrm{i}\left(q_{1}-k_{1}\right) d_{1}}} \mathrm{e}^{\mathrm{i} q_{1}\left(x+b_{1}\right)}+\mathrm{e}^{-\mathrm{i} q_{1}\left(x+b_{1}\right)}-\left(1-\mathrm{e}^{-\mathrm{i}\left(q_{1}+k_{1}\right) d_{1}}\right) \mathrm{e}^{\mathrm{i} q_{1}\left|x+b_{1}\right|}\right], \\
& \Psi_{k_{1}}^{(3)}(x)=A_{k_{1}}\left[\frac{1-\mathrm{e}^{-\mathrm{i}\left(q_{1}+k_{1}\right) d_{1}}}{1-\mathrm{e}^{-\mathrm{i}\left(q_{1}-k_{1}\right) d_{1}}} \mathrm{e}^{\mathrm{i} q_{1}\left(x-L-b_{1}\right)}+\mathrm{e}^{-\mathrm{i} q_{1}\left(x-L-b_{1}\right)}-\left(1-\mathrm{e}^{-\mathrm{i}\left(q_{1}+k_{1}\right) d_{1}}\right) \mathrm{e}^{\mathrm{i} q_{1}\left|x-L-b_{1}\right|}\right] .
\end{aligned}
$$


Let us consider the second layer. For the layer near a first atom we have

$$
f_{1}=A_{1} \mathrm{e}^{-\mathrm{i} q_{2}\left(x-b_{2}\right)}+A_{2} \mathrm{e}^{\mathrm{i} q_{2}\left(x-b_{2}\right)}+B_{1} \mathrm{e}^{\mathrm{i} q_{2}\left|x-b_{2}\right|}, \quad 0 \leqslant x \leqslant b_{2}+\frac{1}{2} d_{2} .
$$

From the condition of discontinuity of the derivative at $x=b_{2}$ we obtain the following relation between the coefficients: $B_{1}=-\frac{U_{2}}{U_{2}+i q_{2} d_{2}}\left(A_{1}+A_{2}\right)$.

The electron wave function in the region of the $N$ th cell is written as follows:

$$
\begin{gathered}
f_{N}=A_{2 N-1} \mathrm{e}^{-\mathrm{i} q_{2}\left(x-b_{2}-(N-1) d_{2}\right)}+A_{2 N} \mathrm{e}^{\mathrm{i} q_{2}\left(x-b_{2}-(N-1) d_{2}\right)}+B_{N} \mathrm{e}^{\mathrm{i} q_{2}\left|x-b_{2}-(N-1) d_{2}\right|}, \\
b_{2}+\frac{2 N-3}{2} d_{2} \leqslant x \leqslant L
\end{gathered}
$$

where $B_{N}=-\frac{U_{2}}{U_{2}+i q_{2} d_{2}}\left(A_{2 N-1}+A_{2 N}\right)$.

To find the wave function of the whole second layer, we use the following matching conditions:

$$
\begin{aligned}
& f_{1}\left(x=b_{2}+\frac{d_{2}}{2}\right)=f_{2}\left(x=b_{2}+\frac{d_{2}}{2}\right), \\
& f_{1}^{\prime}\left(x=b_{2}+\frac{d_{2}}{2}\right)=f_{2}^{\prime}\left(x=b_{2}+\frac{d_{2}}{2}\right), \\
& \ldots \ldots \ldots \ldots \ldots \ldots \ldots \ldots \ldots \ldots \ldots \ldots \ldots \ldots \ldots \ldots \ldots \ldots \ldots \ldots \\
& f_{N-1}\left(x=b_{2}+\frac{2 N-3}{2} d_{2}\right)=f_{N}\left(x=b_{2}+\frac{2 N-3}{2} d_{2}\right), \\
& f_{N-1}^{\prime}\left(x=b_{2}+\frac{2 N-3}{2} d_{2}\right)=f_{N}^{\prime}\left(x=b_{2}+\frac{2 N-3}{2} d_{2}\right),
\end{aligned}
$$

where $N$ is the number of atoms in the second layer. The afore-mentioned matching conditions permit us to determine the following recurrent relations between the coefficients:

$$
A_{2 N-1}=\frac{d_{1}}{d_{5}} A_{2 N-3}+\frac{d_{2}}{d_{5}} A_{2 N-2} ; \quad A_{2 N}=\frac{d_{3}}{d_{5}} A_{2 N-3}+\frac{d_{4}}{d_{5}} A_{2 N-2}
$$

where

$$
\begin{aligned}
d_{1} & =\varphi_{1}\left(d_{2} / 2\right) \varphi_{1}^{\prime}\left(-d_{2} / 2\right)-\varphi_{2}\left(-d_{2} / 2\right) \varphi_{2}^{\prime}\left(d_{2} / 2\right), \\
d_{2} & =\varphi_{2}\left(d_{2} / 2\right) \varphi_{2}^{\prime}\left(-d_{2} / 2\right)-\varphi_{2}\left(d_{2} / 2\right) \varphi_{2}^{\prime}\left(-d_{2} / 2\right), \\
d_{3} & =\varphi_{1}\left(-d_{2} / 2\right) \varphi_{1}^{\prime}\left(d_{2} / 2\right)-\varphi_{1}\left(d_{2} / 2\right) \varphi_{2}^{\prime}\left(-d_{2} / 2\right), \\
d_{4} & =\varphi_{1}\left(-d_{2} / 2\right) \varphi_{2}^{\prime}\left(d_{2} / 2\right)-\varphi_{2}\left(d_{2} / 2\right) \varphi_{1}^{\prime}\left(-d_{2} / 2\right), \\
d_{5} & =\varphi_{1}\left(-d_{2} / 2\right) \varphi_{1}^{\prime}\left(d_{2} / 2\right)-\varphi_{1}\left(d_{2} / 2\right) \varphi_{1}^{\prime}\left(-d_{2} / 2\right), \\
\varphi_{1}(x)=\mathrm{e}^{-\mathrm{i} q_{2}\left(x-b_{2}\right)}- & \frac{U_{2}}{U_{2}+\mathrm{i} q_{2} d_{2}} \mathrm{e}^{\mathrm{i} q_{2}\left|x-b_{2}\right|}, \quad \varphi_{2}(x)=\mathrm{e}^{\mathrm{i} q_{2}\left(x-b_{2}\right)}-\frac{U_{2}}{U_{2}+\mathrm{i} q_{2} d_{2}} \mathrm{e}^{\mathrm{i} q_{2}\left|x-b_{2}\right|} .
\end{aligned}
$$

In order to define the reflection coefficient amplitude $r$ in matching conditions $(7)$ it is necessary to take into account that $f_{i}(x)(i=1,2, \ldots, N)$ are the pieces of the function $\Psi_{2}(x)$, hence $\Psi_{2}(0)=$ $f_{1}(0)$, and $\Psi_{2}(L)=f_{N}(L)$. 


\title{
References
}

1. Karavaev F., Chernyshov V., Fiz. Tekh. Poluprovodn., 2001, 35, 105.

2. Morozova E., Makarovsky O., et al., Fiz. Tekh. Poluprovodn., 2005, 39, 573.

3. Vdovin E., Khanin Yu., Fiz. Tekh. Poluprovodn., 2005, 39, 445.

4. Foreman B.A., Phys. Rev. B, 2007, 76, 045327.

5. Harrison W.A., Phys. Rev., 1961, 123, 85.

6. Grinberg A., Luryi S., Phys. Rev. B, 1989, 39, 7468.

7. Levi A.F.J., Chiu T.H., Solid State Electron., 1988, 31, 625.

8. Trzeciakowski W., Phys. Rev. B, 1988, 38, 12493.

9. Ben Daniel D.J., Duke C.B., Phys. Rev., 1966, 153, 683.

10. Mohan P., Motohisa J., Fukyi T., Appl. Phys. Lett., 2006, 88, 133105.

11. Motohisa J., Noborisaka J., Takeda J., Inari M., Fukyi T., J. Cryst. Growth, 2004, 272, 180.

12. Holovatsky V., Gutsul V., Makhanets O., Rom. J. Phys., 2007, 52, 305.

13. Tkach M., Makhanets O., Gryshuk G., J. Phys. Stud., 2006, 10, 227.

14. Bastard G., Phys. Rev. B, 1981, 24, 5693.

15. Zhu Q.G., Kroemer H., Phys. Rev. B, 1983, 27, 3519.

16. Altarelli M., Phys. Rev. B, 1983, 28, 842.

17. Zhang X., Liu Z., Ip K.M., Leung Y. P., Li Quan, Hark S. K., J. Appl. Phys., 2004, 95, 5752.

18. Orlov L., Ivina N., Fiz. Tverd. Tela, 2004, 46, 913.

19. Han S., Jin W., Zhang D., Tang T., Li Chao, Liu X., Liu Z., Lei Bo, Zhou Ch., Chem. Phys. Lett., 2004, 389, 176.

20. Solanki R., Huo J., Freeouf J.L., Appl. Phys. Lett., 2002, 81, 3864.

21. Vakarchuk I. Kvantova Mekhanika. Lviv University, Lviv, 2004 (in ukrainian).

22. Vukmirovic N., Indjin D., Jovanovic V.D., Ikonic Z., Harrison P. Preprint arXiv: cond-mat/0505607, 1,1 .

23. Boichuk V.I., Bilynski I.V., Fiz. Tverd. Tela, 1995, 37, 734.

24. Baz A.I., Zeldovych Ya.B., Perelomov A.M. Dispersion, reaction and Decays in Irrelativistic Mechanics. Nauka, Moscow, 1971, 544 p. (in russian).

\section{Властивості заряду в наногетеросистемах 3 квантовими ямами та бар'єрами}

\author{
В.І. Бойчук, І.В. Білинський, І.О. Шаклеїна, В.Б. Гольський \\ Кафедра теоретичної фізики, Дрогобицький державний педагогічний університет ім. І. Франка, \\ вул. Франка, 24, Дрогобич, 82100
}

\begin{abstract}
На основі моделі Кроніга-Пенні з $\delta$-подібними потенціалами досліджено тришарову наногетероструктуру, в якій біля меж поділу існує перехідний шар, відстань між крайніми атомами якого $€$ параметром задачі і може змінюватись від нуля до двох сталих ґраток кристалів. Отримана точна формула для коефіцієнта відбивання $R$, з якої можна визначити залежність коефіцієнта від хвильового вектора, величини перехідного шару та величини квантової ями (бар'єру) гетероструктури. Конкретні обчислення проведені для гетеросистем GaAs/AlAs/GaAs та AlAs/GaAs/AlAs. Проведено порівняння коефіцієнтів відбивання у наближенні огинаючих та у методі ефективної маси при малих значеннях хвильового вектора; показано, що близькі результати можна отримати, якщо використати граничні умови Гарісона при відповідним чином підібраних параметрах. Для косинусоїдальної залежності енергії від хвильового вектора, яка виникає в моделі Кроніга-Пенні, в рамках методу Sматриці розсіяння визначено енергію зв'язаних станів для гетероструктури з квантовою ямою (типу $\mathrm{AlAs} / \mathrm{GaAs} / \mathrm{AlAs})$.
\end{abstract}

Ключові слова: гетероструктура, перехідний шар, модель Кроніга-Пенні, коефіцієнт відбивання, S-матриця розсіяння 
